\title{
Does Right-Sided Language Lateralization on BOLD-fMRI Affect Postoperative Language Outcome for AVM Patients?
}

\author{
Zhi LIU1,2, Xiaofeng DENG ${ }^{1}$, Yong CAO ${ }^{1}$, Yuanli ZHAO'1, Jizong ZHAO'1, Shuo WANG ${ }^{1}$ \\ ${ }^{1}$ Department of Neurosurgery, Beijing Tiantan Hospital, Capital Medical University; China National Clinical Research Center for Neurological \\ Diseases (NCRC-ND); Center of Stroke, Beijing Institute for Brain Disorders; Beijing Key Laboratory of Translational Medicine for \\ Cerebrovascular Disease, Beijing, China \\ ${ }^{2}$ ChinaMeitan General Hospital, Department of Neurosurgery, Beijing, China
}

\section{ABSTRACT}

AIM: For cerebral arteriovenous malformations (AVMs) involving language areas, right-sided language lateralization on functional magnetic resonance imaging (fMRI) has been reported, which is regarded as language cortex reorganization. The authors attempted to study if this right-sided language lateralization affects postoperative language outcome.

MATERIAL and METHODS: Clinical and imaging data of 43 right-handed AVM patients who underwent preoperative fMRI were retrospectively reviewed. All lesions involved the language cortex, with the Broca area involved in 13 patients and the Wernicke area involved in 30 patients. Lateralization indices (LI) of BOLD signal activations were calculated to determine language lateralization. All patients underwent craniotomy and total resection. Western aphasia battery (WAB) was used to evaluate language functions preoperatively, 1-2 weeks after surgery and 6-30 months after surgery.

RESULTS: On preoperative fMRI, right-sided lateralization was observed in 18 patients (41.9\%, R Group), including 3 with rightsided lateralization in the Broca area alone, 14 in the Wernicke area alone, and 1 in both areas. The other 25 patients were non-rightsided lateralized (NR Group). One week after surgery, 7 patients in the R Group (38.9\%) and 11 patients in the NR Group (44.0\%) had language function deterioration, and no significant difference was found $(p=0.983)$. At long-term follow-up, 3 patients in the R Group (16.7\%) and 4 patients in the NR Group (16.0\%) still had aphasia, and no significant difference was observed ( $p=1.000)$.

CONCLUSION: Although right-sided lateralization on $\mathrm{fMRI}$ might suggest language cortex reorganization, it is not a factor predicting better postoperative language outcome for AVM patients.

KEYWORDS: Cerebral arteriovenous malformation, Language cortex reorganization, Functional magnetic resonance imaging, Aphasia

\section{INTRODUCTION}

$\mathrm{C}$ erebral arteriovenous malformations (AVMs) are congenital vascular lesions. The pathological characterisare direct communication of arteries to abnormal veins without interposing capillaries $(7,20)$. An interesting clinical phenomenon is that although AVMs may grow in the language cortex, they usually do not present with aphasia unless ruptured, as reported in our previous study, which focused on the preoperative blood oxygen level-dependent (BOLD) functional magnetic resonance imaging (fMRI) of AVM patients (9). Our previous study also showed that for cerebral AVMs involving language areas at traditional anatomical locations, right-sided lateralization of BOLD signals is not rare (36.5\%) (9). Both of these phenomenons are considered as language cortex reorganization $(1,9)$. 
In our opinion, a better language outcome after AVM resection surgery might be expected for patients with right-sided lateralization of BOLD signals, because the language areas are thought to have transferred to the homologous regions of the right hemisphere and language function will therefore be seldom impaired by the surgery.

In this study, we retrospectively reviewed clinical and imaging data of 43 right-handed cerebral AVM patients who underwent BOLD fMRI studies, with all lesions located in the left hemisphere and involved language areas. Total resection under neuronavigation was performed on all patients. Language functions were evaluated preoperatively and postoperatively with the Western Aphasia Battery (WAB). The focus of this study was to demonstrate if right-sided language lateralization on preoperative $\mathrm{fMRI}$ affects postoperative language outcome.

\section{MATERIAL and METHODS}

\section{Patient Demographics}

This study was approved by the Institutional Review Board (IRB) of Beijing Tiantan hospital, Capital Medical University, and all participating patients gave consent. We retrospectively reviewed the medical records and imaging studies of 43 cerebral AVMs patients, who underwent fMRI for preoperative evaluation between January 2013 and April 2015. Selection criteria were as follows: 1 . all patients were right-handed, demonstrated by the Edinburgh handedness inventory (8); 2. all lesions were located in the left hemisphere and involved the language cortex, including the Broca (inferior frontal and middle frontal gyri, including Brodmann area 44, 45, 9, 46) and Wernicke (supramarginal, angular, and superior temporal gyri, including Brodmann area 22, 21, 39, 40) areas (5); 3. patients underwent surgical resection under neuronavigation. None of these patients were included in our previous study (9).

According to the criteria, as shown in Table I, 43 patients were enrolled in this study, including 31 males and 12 females. Ages ranged from 11 to 55 years with an average of $29.9 \pm 12.5$ years. Main clinical presentations included hemorrhage in 17 patients (39.5\%), seizures in 15 (34.9\%), headache in $6(20.0 \%)$, dizziness in $3(7.0 \%)$, aphasia in 1 $(2.3 \%)$, and asymptomatic in 1 patient $(2.3 \%)$. Two patients had undergone stereotactic radiosurgery (SRS) and 1 patient endovascular embolization. Thirteen patients' lesions involved the Broca area, and the other 30 patients' lesions involved the Wernicke area. The mean diameter of the AVMs was 41.4 $\mathrm{mm}$ (range, 9.8-82.9 mm). According to Spetzler-Martin (S-M) grading system, there were 7 grade II patients, 30 grade III patients and 6 grade IV patients $(7,20)$.

\section{fMRI Acquisition}

A Siemens Medical Systems Trio 3.0 T MRI system equipped at the $306^{\text {th }}$ hospital of People's Liberation Army was used for fMRI signal acquisition, which was performed approximately 1-2 weeks before surgery. Every patient underwent a localizing image scan followed by a T2 structure image scan; then a T2-weighted functional image was obtained (T2-weighted gradient echo, echo-planner imaging, $\mathrm{TR}=3000 \mathrm{~ms}, \mathrm{TE}=30$ $\mathrm{ms}, \mathrm{FOV}=220 \times 220 \mathrm{~mm}^{2}$, flip angle $=9^{\circ}$, matrix $=64 \times 64$, slice thickness $=1.0 \mathrm{~mm}$, voxel size $=1.0 \times 1.0 \times 1.0 \mathrm{~mm}^{3}$ ); finally, a T1-weighted three-dimensional fine structure image was scanned $\left(T R=2300 \mathrm{~ms}\right.$, TE $=2.98 \mathrm{~ms}$, flip angle $=9^{\circ}$, FOV $=256 \times 256 \mathrm{~mm}^{2}$, matrix $=64 \times 64$, slice thickness $=1.0$ $\mathrm{mm}$, voxel size $=1.0 \times 1.0 \times 1.0 \mathrm{~mm}^{3}$ ).

Informed consent complying with local IRB regulations was obtained from all patients. Block-designed fMRI was performed and patients were tested with two lexical-semantic language paradigms: picture naming and verb generation. During picture naming, different pictures were visually presented to the center of the patient's visual field. The patient was instructed to silently name each object as it appeared. In the control condition, the patient was instructed to passively view a picture of chopsticks. During verb generation, common concrete (written) nouns were presented to the center of the patient's visual field. The patient was instructed to silently generate a verb associated with each noun. In the control condition, the patient passively viewed a pound sign (\#). During each paradigm, a set of 40 tasks was divided equally into 8 performance periods of 5 tasks each and interleaved with 8 control condition periods. Tasks were presented at a rate of one every 3 seconds. Before scanning, all patients were trained with the paradigms using stimuli different from those presented during the imaging protocol, and all of them demonstrated the ability to perform the tasks.

\section{Image Processing}

fMRI data was processed on a Matlab 8.4 workstation (Mathworks, Natick, Massachusetts) with SPM 8 (London University) and xjView toolbox (Stanford University). Voxels that exceeded a predetermined significance threshold $(p<0.001)$ were overlaid on the T1-weighted structure images to display the anatomical location of brain activity (9).

The method determining language lateralization was concordant with our previous study (9). Language lateralization was studied in the Broca and Wernicke areas separately (24). The Broca area refers to the inferior frontal and middle frontal gyri, including Brodmann area 44, 45, 9, 46. The Wernicke area is defined as the supramarginal, angular, and superior temporal gyri, including Brodmann area 22, 21, 39, 40. The lateralization index (LI) was used to quantify the degree of lateralization of BOLD signal activations: $L I=(V L-V R) /(V L+V R)$, where VL denotes the number of voxels activated in the left hemisphere and VR is the number of voxels activated in the right hemisphere. Language activation was categorized into 3 patterns. An LI less than or equal to -0.2 was defined as right-sided lateralization of BOLD signal activations ( $R$ Group), whereas an $\mathrm{LI}$ greater than or equal to $0.2 \mathrm{~s}$ was considered as left-sided lateralization. An LI between -0.2 and 0.2 was regarded as no clear hemispheric preference (17). In this study, patients with left-sided lateralization or no clear hemispheric preference were both categorized in the non-right group (NR Group).

\section{Treatment}

fMRI data was further processed on a Brainlab workstation and used for intraoperative neuronavigation. All the 43 AVM 
patients were surgically treated and total resection was achieved in all patients, which was confirmed by postoperative digital subtraction angiography (DSA). No patient underwent Wada test or intraoperative electrocortical stimulation mapping (ESM) $(3,27)$.

\section{Language Function Evaluation and Follow-up}

Language function was evaluated with WAB before surgery, at short-term follow-up (1-2 weeks after surgery), and at longterm follow-up (6-30 months after surgery) (10).

\section{Statistical Analysis}

All statistical analyses were performed with SPSS (Windows version 18.0, IBM). Chi-square test for the $R \times C$ contingency tables was used to analyze the difference of language
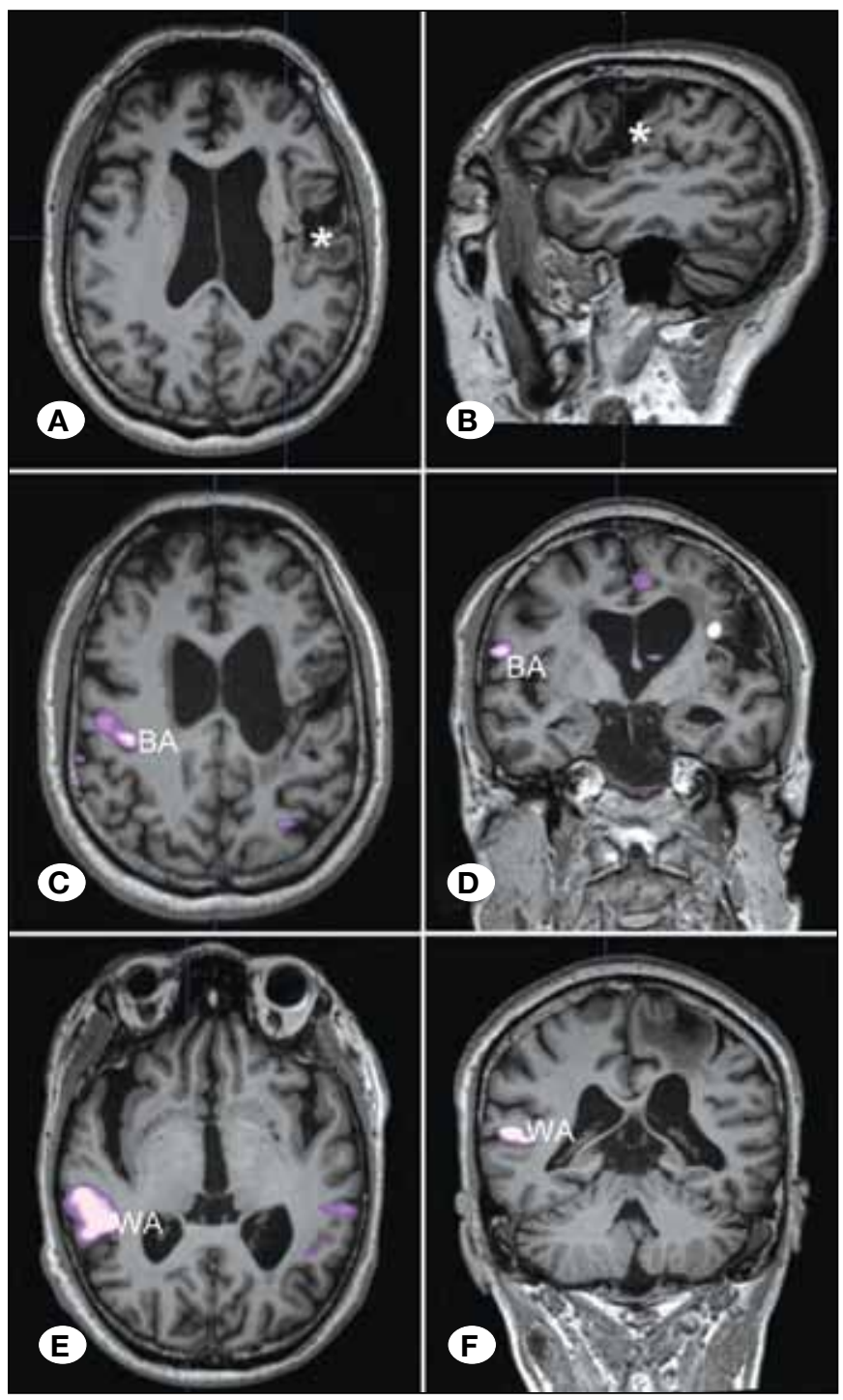

Figure 1: $\mathrm{MRI}$ of a patient in R Group, with lesions involving the Broca area (case 4). T1-weighted axial (A) and sagittal (B) MR images revealed an AVM (*) involving the Broca area. $\mathrm{fMRI}$ images showed right-sided lateralization of both the Broca (C, D: BA) and Wernicke (E, F: WA) areas. dysfunction between groups. A probability value $<0.05$ was considered as statistically significant.

\section{RESULTS}

\section{Clinical and Imaging Characteristics}

Twelve males and 6 females, with ages ranging from 20 to 55 years (mean $29.9 \pm 12.5$ years) were enrolled in the $R$ Group. Four patients had lesions involving the Broca area (Figure 1A$\mathrm{F})$ and 14 patients had lesions involving the Wernicke area (Figure 2A-F). Lesion size ranged from 19.5 to $66.1 \mathrm{~mm}$ (mean $39.1 \pm 11.8 \mathrm{~mm}$ ). There were $3 \mathrm{~S}-\mathrm{M}$ grade II patients, 14 grade III patients, and 1 grade IV patient.

Meanwhile, 19 males and 6 females, with ages ranging from 11 to 53 years (mean 26.5 \pm 12.6 years) were enrolled in the NR Group. Nine patients had lesions involving the Broca area and 16 patients had lesions involving the Wernicke area. The average lesion size was $43.1 \pm 18.3 \mathrm{~mm}$ (range, 9.8-82.9 mm). There were 4 grade II patients, 16 grade III patients, and 5 grade IV patients.

Statistical results showed that there was no significant difference between the two groups in gender (c2: 0.453 , $p=0.501)$, age $(p=0.926$, by independent t-test), lesion location (c2: $0.942, p=0.332)$, lesion size $(p=0.062$, by independent $t$ test) or S-M grades ( $p=0.386$, by Mann-Whitney test).

\section{fMRI data analysis}

According to the above-mentioned criteria, as shown in Table I, right-sided lateralization was observed in 18 patients $(41.9 \%$, $\mathrm{R}$ Group), including 3 with right-sided lateralization in the Broca area alone, 14 in the Wernicke area alone, and 1 in both areas. The other 25 patients were non-right-sided lateralized (NR Group), including 22 patients with left-sided lateralization and 3 patents with no clear hemispheric preference.

\section{Language Function Evaluation and Follow-up}

Postoperatively, all patients were followed up. The follow-up duration ranged from 6 to 30 months, with an average period of 18 months. The language functions according to the WAB tests were summarized in Table I.

\section{Preoperative Evaluation}

Before surgery, 2 patients presented with anomic aphasia after intracranial hemorrhage (cases 7 and 29). The other patients' language functions were normal. Although the initial presentation of case 6 was transient aphasia, his language function recovered to normal at admission.

\section{Short-term Outcome (1-2 weeks after surgery)}

In the $\mathrm{R}$ Group, 11 patients maintained their language status $(61.1 \%)$, including case 7 , who had anomic aphasia both before and after surgery. Seven patients' (38.9\%) language function deteriorated in the short-term postoperative period, including 6 patients with anomic aphasia, 1 with motor aphasia, and 1 with conduction aphasia. In the NR Group, 14 patients' language status was stable (56.0\%), including case 29 , who had anomic aphasia both before and after surgery. 
Language function deterioration was observed in 11 patients (44.0\%), including 6 patients with anomic aphasia, 4 with conduction aphasia, and 1 with transcortical motor aphasia. Although the incidence of language function deterioration was higher in the NR Group (44.0\%) than in the R Group (38.9\%), no significant difference was observed (c2: $0.112, p=0.738)$.

\section{Long-term Outcome (6-30 months after surgery, the most recent follow-up)}

As shown in Table I, in the R Group, 3 patients (16.7\%) still had aphasia (including 2 with conduction aphasia and 1 with anomic aphasia), and the other 5 patients restored their language function. In the NR Group, 4 patients (16.0\%) still had aphasia (including 3 with anomic aphasia and 1 with conduction aphasia), the other 8 patients' language functions returned to normal. Likewise, no significant difference of aphasia was observed between the R Group and the NR Group (c2: 0.000, $p=1.000$ ).

\section{- DISCUSSION}

\section{Left Hemisphere Language Dominance and Its Challenge}

One major question in neuroscience is how each hemisphere contributes to language processing, as the most confusing morphological characteristic of the human brain is that the two cerebral hemispheres are seemingly symmetrical. The left hemisphere language dominance for right-handed human was recognized at the end of the $19^{\text {th }}$ century. A patient who had lost the ability to speak was referred to a French surgeon Paul Broca. After autopsy, Broca found a lesion in the left inferior frontal gyrus (IFG) and associated this region with the capacity for articulate speech (13). In addition, supported by Wernicke's association of the sound of words with the left posterior superior temporal gyrus (STG) and Dejerine's recognition of the acquired inability to read and write following left angular gyrus damage, a dominant role of the left hemisphere in language processing for right-handed human was established $(4,19)$.

The neurological axiom of exclusive left hemisphere dominance in language was fundamentally challenged by behavioral studies with callosotomy patients. In the "split-brain" syndrome, unilateral sensory input is transmitted to the contralateral hemisphere, where, due to the lack of interhemispheric connections, it cannot be shared with the opposite hemisphere. For example, language stimuli transmitted to the right hemisphere were not available for processing in the left hemisphere. Therefore, the role of the right hemisphere in language has been gradually recognized, no matter in healthy population or in aphasia patients $(12,26)$.

\section{Modalities for Assessment of Language Dominance}

Clinically, there are several techniques for judging language dominance and language mapping, including intra-arterial Amytal injection (Wada test), ESM, fMRI, Magnetoencephalography (MEG), and radionuclide scanning (SPECT and PET). The Wada test has conventionally been recognized as the gold standard for assessment of language dominance. It is, however, invasive and does not provide the topographic specificity offered by fMRI. fMRI has been widely used for pre- operative language mapping and the assessment of language lateralization, as it is noninvasive and offers topographic maps of language function with very high sensitivity and acceptable specificity $(6,17)$.

Some authors have casted questions on the accuracy of fMRI, especially in AVM patients. The hypothesized theory is that AVMs could reduce the cerebral perfusion around extranidal tissues (the so called "arterial steal"), resulting in abnormal fMRI results, as BOLD fMRI is dependent on blood oxygen levels (30). However, until recently, a number of studies compared fMRI and the Wada test or ESM, and the results have demonstrated that even in patients with vascular malformations, fMRI is an excellent method for language lateralization $(5,14,22)$.
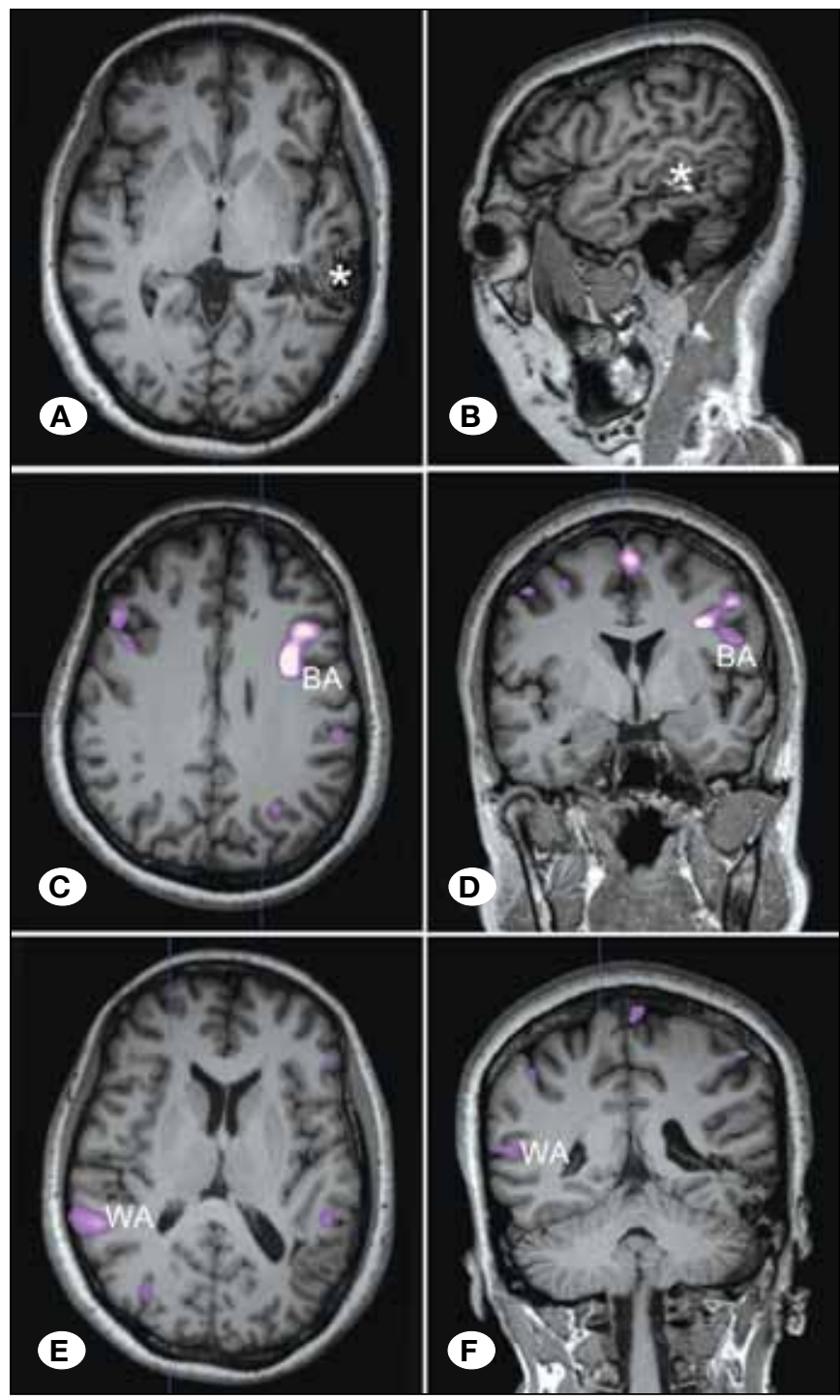

Figure 2: $M R I$ of a patient in R Group, with lesions involving the Wernicke area (case 8). T1-weighted axial (A) and sagittal (B) MR images revealed an AVM $\left({ }^{*}\right)$ involving the Wernicke area. $\mathrm{fMRI}$ images demonstrated left-sided lateralization of the Broca area (C, D: BA) and right-sided lateralization of the Wernicke area (E, F: WA). 

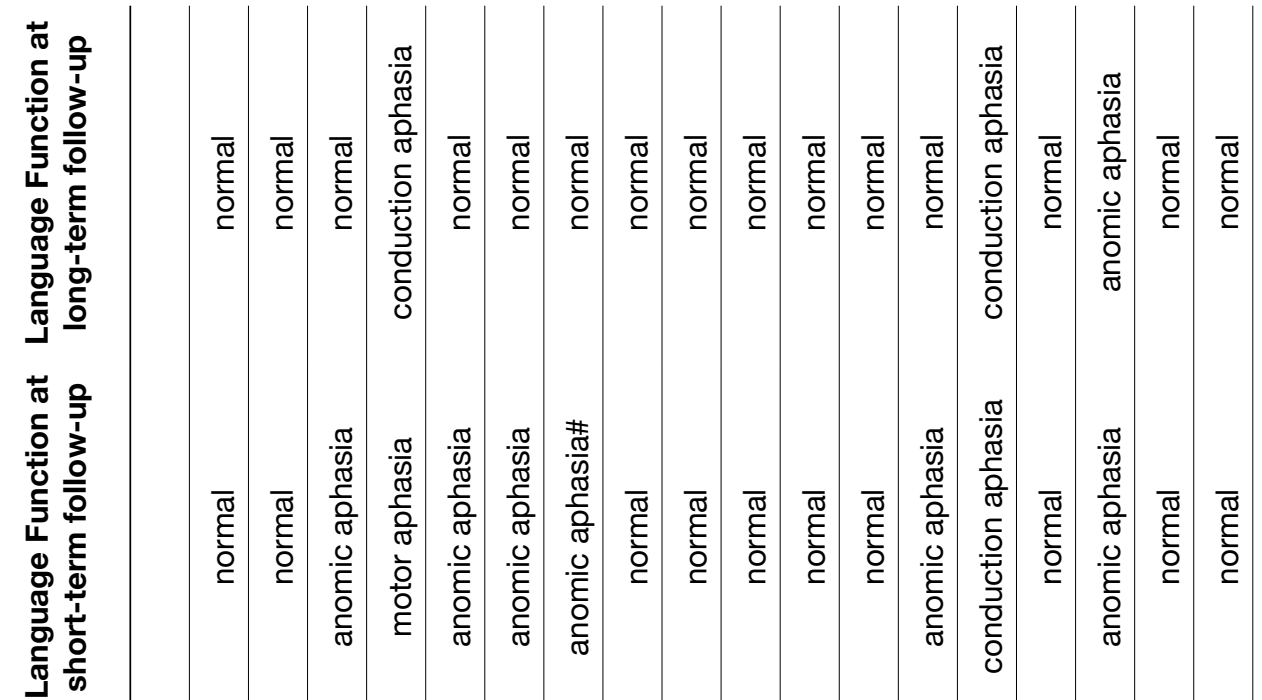

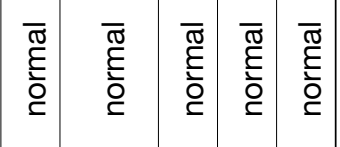

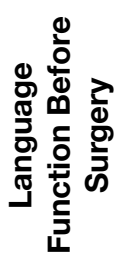

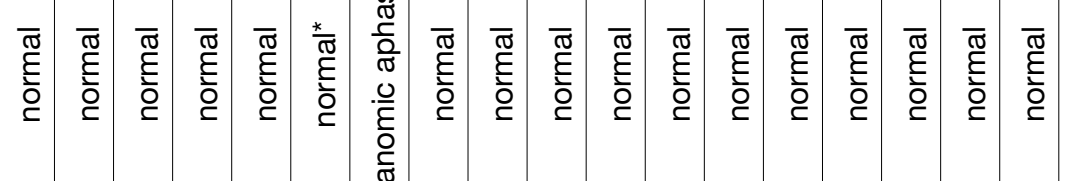
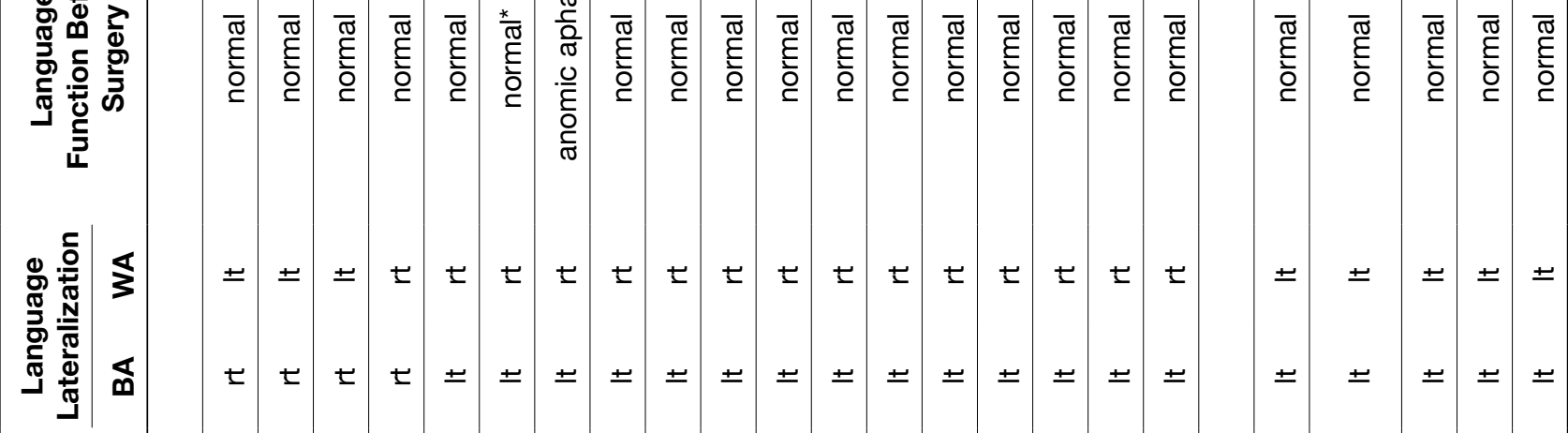

$\pm \pm E E t E E t E E t E t E t E t$

$\pm \quad \pm \pm \pm$

$t \pm t \pm \pm \pm \pm \pm \pm \pm \pm \pm \pm \pm \pm \pm \pm$

$\pm \pm \pm \pm \pm$

竞离

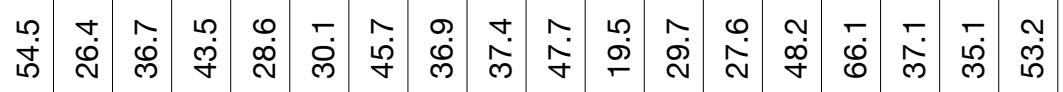

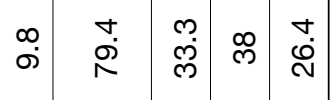

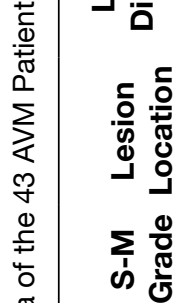

茜

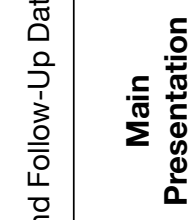

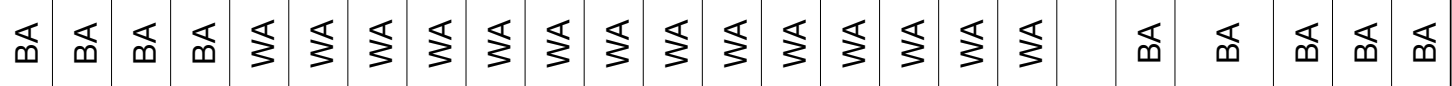

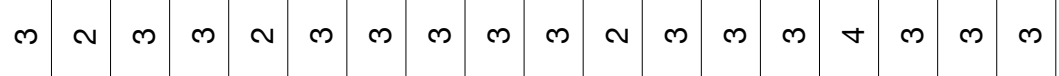

$\sim+\infty \infty \infty$

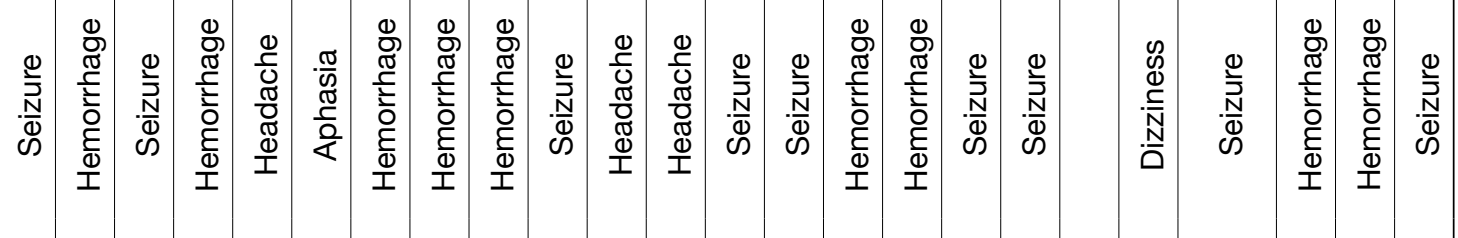

$\stackrel{\times}{\oplus}$

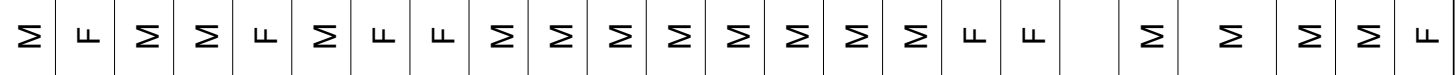

焉旁

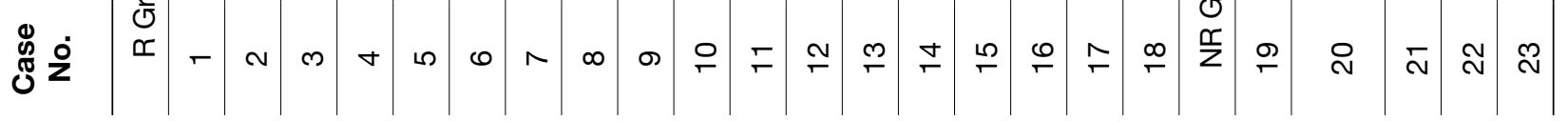




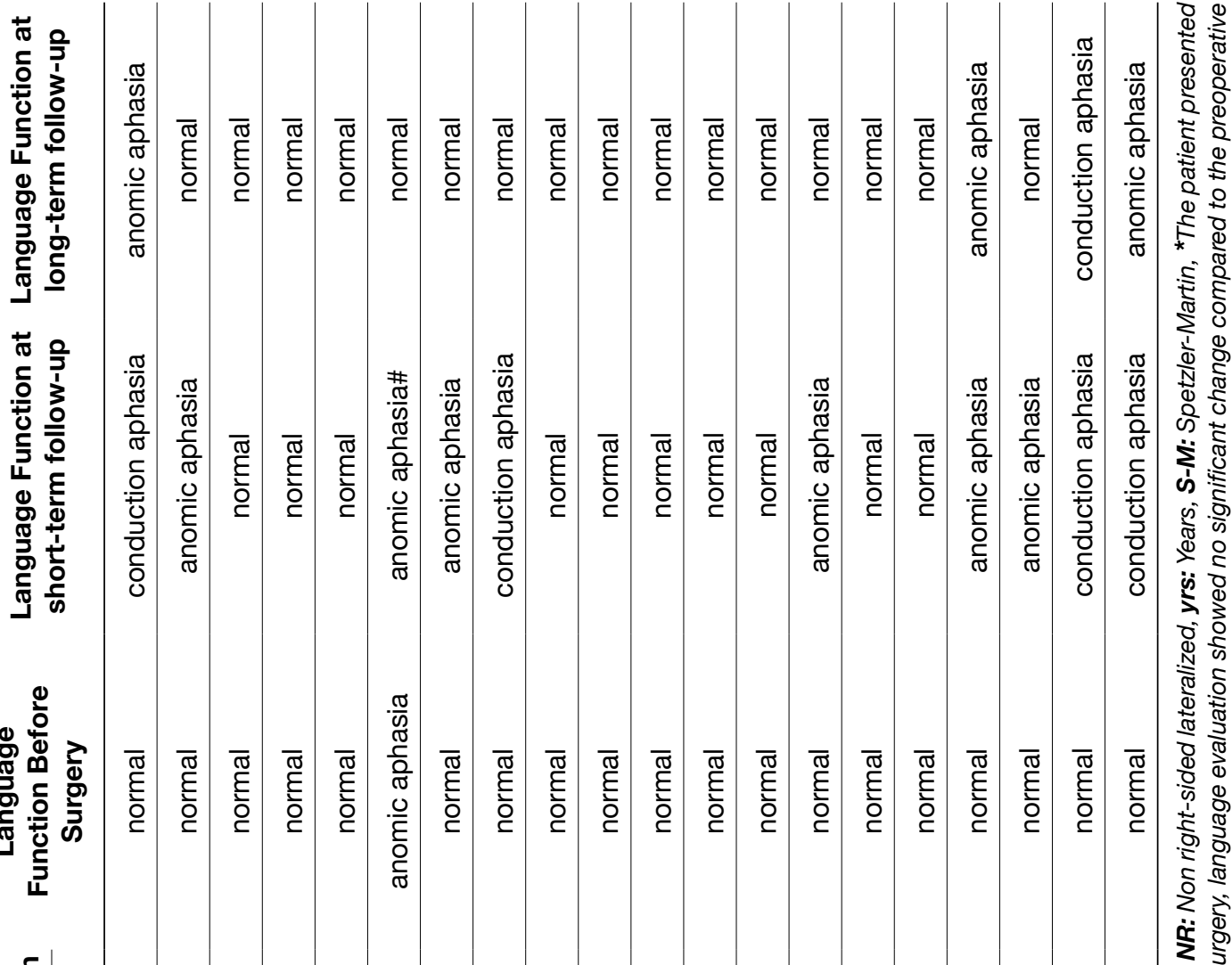

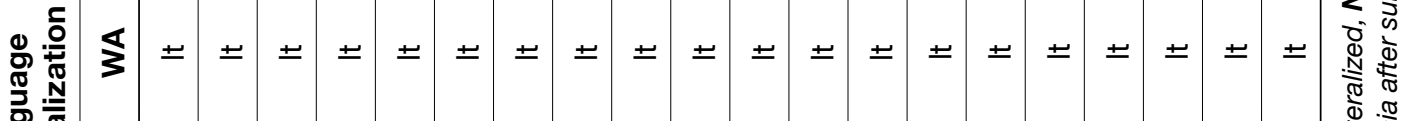

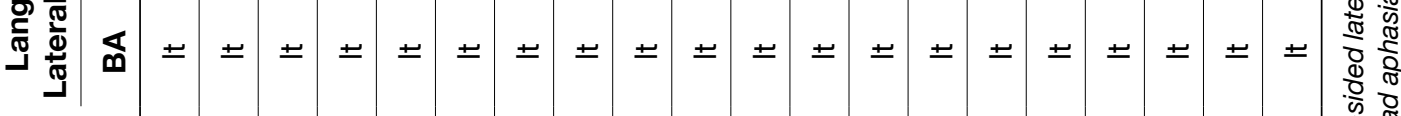

ᄃ

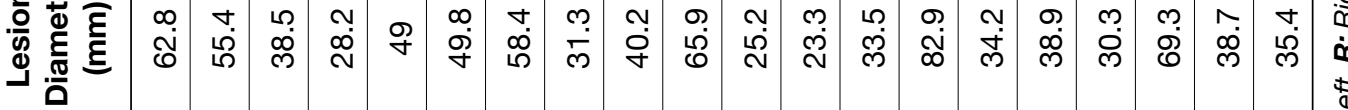

西染

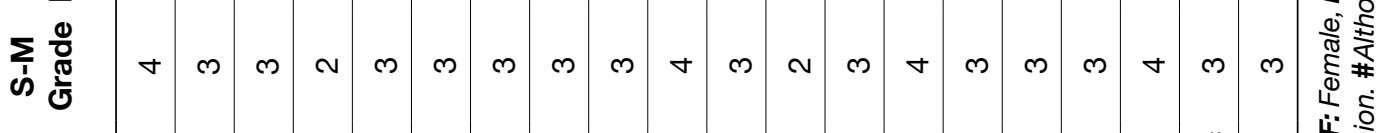

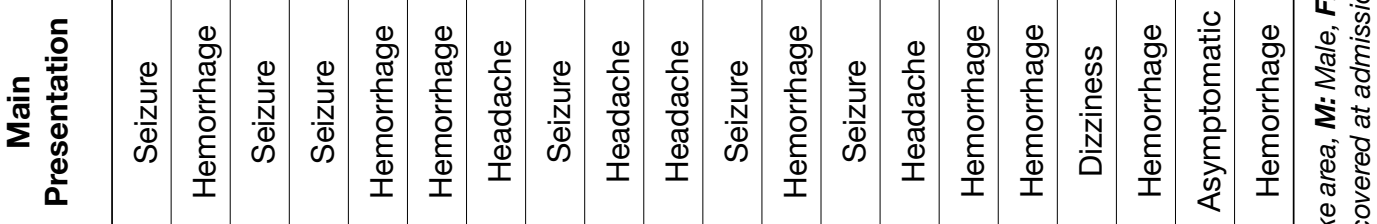

Ф

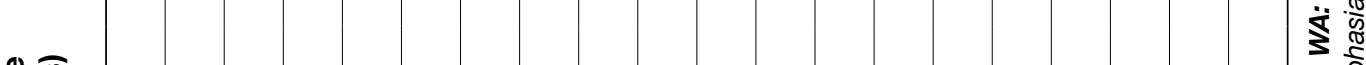

ن்

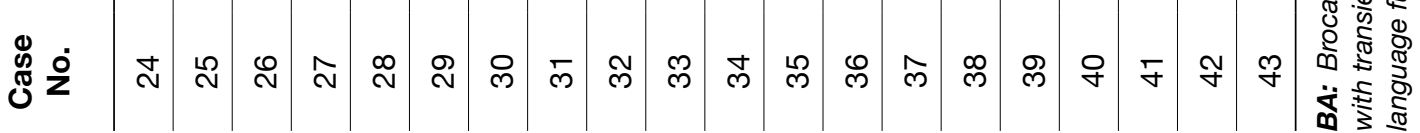




\section{The Clinical Significance of Right-sided Language Lateralization}

Previous studies have shown language reorganization in the setting of various intracranial diseases, including AVMs $(1,16,18,32)$, strokes $(21,34)$, brain tumors $(11,23,25,33)$, epilepsies $(2,15)$, etc. The language reorganization in AVM patients has been of particular interest because it has been noticed that unruptured AVMs located in the language cortex usually do not lead to aphasia. In our previous study, 63 AVM patients were included and only 3 patients presented with language disorder (4.8\%) (9). In this series, only 1 AVM patient without intracranial hemorrhage presented with transient aphasia before surgery $(2.3 \%)$. This is different from other intracranial disorders. For example, for brain tumors involving the language areas, language dysfunction is a very common symptom.

Right-sided dominance is not a very rare phenomenon for AVM patients with lesions involving the language cortex $(9,17)$, and some studies have shown that the right hemisphere might be also very important in language processing $(28,29,31)$. In our previous study, 23 of the 63 patients showed rightsided lateralization (36.5\%), which was considered as suggesting language cortex reorganization (9). However, our previous study focused on the preoperative fMRI findings, and postoperative language functions were not evaluated. As a result, the significance of the right-sided lateralization and especially the effect on the language function outcome after surgery on BOLD fMRI are still unclear. Therefore, we conducted this study to evaluate if right-sided lateralization on preoperative $\mathrm{fMRI}$ could lower the incidence of language disorders after surgery. A series of 43 AVM patients with lesions involving language areas were retrospectively reviewed, and the language functions were systematically evaluated before surgery, at short-term follow-up and at long-term follow-up. On preoperative $\mathrm{fMRI}$, right-sided lateralization was observed in 18 patients, and thus the incidence of right-sided lateralization was up to $41.9 \%$. The high incidence might be attributed to that the AVM is a congenital lesion and develops before the period of language learning. When AVMs develop in the anatomical site of eloquent cortex in early life, neuroplasticity will result in cortical reorganization of the functional areas, with displacement to other regions, including the areas around the lesion on the same hemisphere and the homologous regions on the contralateral hemisphere.

However, although this right-sided lateralization in our study suggests language reorganization, follow-up language evaluation showed that there was no significant difference between the R Group and the NR Group, which means that right-sided lateralization on BOLD $\mathrm{fMRI}$ could not decrease the incidence of language dysfunction after surgery. We think there are two explanations for this phenomenon. First, although LI showed right-sided lateralization of these 18 patients, this does not mean there was no activation in the language areas on the left hemisphere. In contrast, BOLD signal activations were also observed in the traditional language areas on the left side, suggesting that these areas are still functional and might even be essential for language function. The language areas on the right side may play only part of language function. Second, although previous studies showed the fMRI had a high concordance with the Wada test in determining language dominance, the results of $\mathrm{AMRI}$ might be unreliable, especially for those patients with atypical lateralization. We did not perform Wada tests in this series because of its invasive character, so the language lateralization cannot be confirmed.

\section{Limitations of Study}

The present study has several limitations. First, although we enrolled a large series of 43 AVM patients with nidus involving language areas, the sample size, might not be big enough to determine the difference of the two groups, which may lead to false-negative findings. Second, this is an fMRI study, and the confirmatory tests, ESM and Wada testing were not conducted.

\section{CONCLUSION}

Right-sided dominance is not a rare phenomenon for AVM patients with lesions involving the language cortex. Although right-sided lateralization suggests language reorganization, it does not decrease the incidence of language dysfunction after surgery. Neurosurgeons still need to pay attention to the language function protection in these patients.

\section{ACKNOWLEDGMENT}

This study received a grant from the Ministry of Science and Technology of China (2011BAI08B08 and 2012CB825505), National Key Technology Research and Development Program of the Ministry of Science and Technology of China (2013BAI09B03), and Center of Stroke, Beijing Institute for Brain Disorders (BIBD-PXM2013_014226_07_000084).

\section{REFERENCES}

1. Alkadhi H, Kollias SS, Crelier GR, Golay X, Hepp-Reymond $\mathrm{MC}$, Valavanis A: Plasticity of the human motor cortex in patients with arteriovenous malformations: A functional MR imaging study. AJNR Am J Neuroradiol 21:1423-1433,2000

2. Baciu M, Perrone-Bertolotti M: What do patients with epilepsy tell us about language dynamics? A review of fMRI studies. Rev Neurosci 26:323-341, 2015

3. Bauer PR, Reitsma JB, Houweling BM, Ferrier CH, Ramsey NF: Can fMRI safely replace the Wada test for preoperative assessment of language lateralisation? A meta-analysis and systematic review. J Neurol Neurosurg Psychiatry 85:581588, 2014

4. Bub DN, Arguin M, Lecours AR: Jules Dejerine and his interpretation of pure alexia. Brain Lang 45:531-559, 1993

5. Cannestra AF, Pouratian N, Forage J, Bookheimer SY, Martin NA, Toga AW: Functional magnetic resonance imaging and optical imaging for dominant-hemisphere perisylvian arteriovenous malformations. Neurosurgery 55:804-812; discussion 812-814, 2004 
6. Chen P, Lin J, Chen B, Lu C, Guo T: Processing emotional words in two languages with one brain: ERP and $\mathrm{fMRI}$ evidence from Chinese-English bilinguals. Cortex 71:34-48, 2015

7. Choi JH, Mohr JP: Brain arteriovenous malformations in adults. Lancet Neurol 4:299-308, 2005

8. Christman SD, Prichard EC, Corser R: Factor analysis of the Edinburgh Handedness Inventory: Inconsistent handedness yields a two-factor solution. Brain Cogn 98:82-86, 2015

9. Deng X, Zhang Y, Xu L, Wang B, Wang S, Wu J, Zhang D, Wang $\mathrm{R}$, Wang J, Zhao J: Comparison of language cortex reorganization patterns between cerebral arteriovenous malformations and gliomas: A functional MRI study. J Neurosurg 122:996-1003, 2015

10. Dickey MW, Warren T: The influence of event-related knowledge on verb-argument processing in aphasia. Neuropsychologia 67:63-81, 2015

11. Duffau $\mathrm{H}$ : The huge plastic potential of adult brain and the role of connectomics: New insights provided by serial mappings in glioma surgery. Cortex 58:325-337, 2014

12. Gold R, Faust M, Ben-Artzi E: Metaphors and verbal creativity: The role of the right hemisphere. Laterality 17:602-614, 2012

13. Hagoort P: Nodes and networks in the neural architecture for language: Broca's region and beyond. Curr Opin Neurobiol 28:136-141, 2014

14. Janecek JK, Swanson SJ, Sabsevitz DS, Hammeke TA, Raghavan M, E Rozman M, Binder JR: Language lateralization by $\mathrm{fMRI}$ and Wada testing in 229 patients with epilepsy: Rates and predictors of discordance. Epilepsia 54:314-322, 2013

15. Janszky J, Mertens M, Janszky I, Ebner A, Woermann FG: Left-sided interictal epileptic activity induces shift of language lateralization in temporal lobe epilepsy: An fMRI study. Epilepsia 47:921-927, 2006

16. La Piana R, Klein D, Cortes M, Tampieri D: Speech reorganization after an AVM bleed cured by embolization. A case report and review of the literature. Interv Neuroradiol 15:456-461, 2009

17. Lee DJ, Pouratian N, Bookheimer SY, Martin NA: Factors predicting language lateralization in patients with perisylvian vascular malformations. Clinical article. J Neurosurg 113:723730, 2010

18. Lehericy S, Biondi A, Sourour N, Vlaicu M, du MST, Cohen L, Vivas E, Capelle L, Faillot T, Casasco A, Le Bihan D, Marsault C: Arteriovenous brain malformations: Is functional MR imaging reliable for studying language reorganization in patients? Initial observations. Radiology 223:672-682, 2002

19. Mesulam MM, Thompson CK, Weintraub S, Rogalski EJ: The Wernicke conundrum and the anatomy of language comprehension in primary progressive aphasia. Brain 138:2423-2437, 2015

20. Mohr JP: Brain arteriovenous malformations: Children and adults. Stroke 36:2060-2061, 2005

21. Otal B, Olma MC, Floel A, Wellwood I: Inhibitory noninvasive brain stimulation to homologous language regions as an adjunct to speech and language therapy in post-stroke aphasia: A meta-analysis. Front Hum Neurosci 9:236, 2015
22. Pouratian N, Bookheimer SY, Rex DE, Martin NA, Toga AW: Utility of preoperative functional magnetic resonance imaging for identifying language cortices in patients with vascular malformations. J Neurosurg 97:21-32, 2002

23. Rosler J, Niraula B, Strack V, Zdunczyk A, Schilt S, Savolainen P, Lioumis P, Makela J, Vajkoczy P, Frey D, Picht T: Language mapping in healthy volunteers and brain tumor patients with a novel navigated TMS system: Evidence of tumor-induced plasticity. Clin Neurophysiol 125:526-536, 2013

24. Rutten GJ, Ramsey NF, van Rijen PC, Alpherts WC, van Veelen CW: FMRI-determined language lateralization in patients with unilateral or mixed language dominance according to the Wada test. Neuroimage 17:447-460, 2002

25. Sanai N, Mirzadeh Z, Berger MS: Functional outcome after language mapping for glioma resection. N Engl J Med 358:1827,2008

26. Spotorno S, Faure S: Change detection in complex scenes: Hemispheric contribution and the role of perceptual and semantic factors. Perception 40:5-22, 2011

27. Stapleton CJ, Walcott BP, Fusco MR, Thomas AJ, Ogilvy CS: Brain mapping for safe microsurgical resection of arteriovenous malformations in eloquent cortex. World Neurosurg 83:11481156, 2015

28. Taylor KI, Regard M: Language in the right cerebral hemisphere: Contributions from reading studies. News Physiol Sci 18:257-261, 2003

29. Turkeltaub PE, Coslett HB, Thomas AL, Faseyitan O, Benson $\mathrm{J}$, Norise $\mathrm{C}$, Hamilton $\mathrm{RH}$ : The right hemisphere is not unitary in its role in aphasia recovery. Cortex 48:1179-1186, 2012

30. Ulmer JL, Hacein-Bey L, Mathews VP, Mueller WM, DeYoe EA, Prost RW, Meyer GA, Krouwer HG, Schmainda KM: Lesion- induced pseudo-dominance at functional magnetic resonance imaging: Implications for preoperative assessments. Neurosurgery 55:569-579; discussion 580-581, 2004

31. Van Ettinger-Veenstra $H$, Ragnehed M, McAllister A, Lundberg $\mathrm{P}$, Engstrom M: Right-hemispheric cortical contributions to language ability in healthy adults. Brain Lang 120:395-400, 2012

32. Vikingstad EM, Cao Y, Thomas AJ, Johnson AF, Malik GM, Welch KM: Language hemispheric dominance in patients with congenital lesions of eloquent brain. Neurosurgery 47:562570,2000

33. Wang L, Chen D, Yang X, Olson JJ, Gopinath K, Fan T, Mao H: Group independent component analysis and functional MRI examination of changes in language areas associated with brain tumors at different locations. PLOS ONE 8:e59657, 2013

34. Winhuisen L, Thiel A, Schumacher B, Kessler J, Rudolf J, Haupt WF, Heiss WD: Role of the contralateral inferior frontal gyrus in recovery of language function in poststroke aphasia: a combined repetitive transcranial magnetic stimulation and positron emission tomography study. Stroke 36:1759-1763, 2005 